This item was submitted to Loughborough's Research Repository by the author.

Items in Figshare are protected by copyright, with all rights reserved, unless otherwise indicated.

\title{
Gaudin subalgebras and wonderful models
}

PLEASE CITE THE PUBLISHED VERSION

http://dx.doi.org/10.1007/s00029-015-0213-y

PUBLISHER

(C) Springer

VERSION

AM (Accepted Manuscript)

PUBLISHER STATEMENT

This work is made available according to the conditions of the Creative Commons Attribution-NonCommercialNoDerivatives 4.0 International (CC BY-NC-ND 4.0) licence. Full details of this licence are available at: https://creativecommons.org/licenses/by-nc-nd/4.0/

\section{LICENCE}

CC BY-NC-ND 4.0

\section{REPOSITORY RECORD}

Aguirre, Leonardo, G. Felder, and Alexander Veselov. 2019. "Gaudin Subalgebras and Wonderful Models". figshare. https://hdl.handle.net/2134/19974. 


\title{
GAUDIN SUBALGEBRAS AND WONDERFUL MODELS
}

\author{
LEONARDO AGUIRRE, GIOVANNI FELDER, AND ALEXANDER P. VESELOV
}

\begin{abstract}
Gaudin Hamiltonians form families of $r$-dimensional abelian Lie subalgebras of the holonomy Lie algebra of the arrangement of reflection hyperplanes of a Coxeter group of rank $r$. We consider the set of principal Gaudin subalgebras, which is the closure in the appropriate Grassmannian of the set of spans of Gaudin Hamiltonians. We show that principal Gaudin subalgebras form a smooth projective variety isomorphic to the De Concini-Procesi compactification of the projectivized complement of the arrangement of reflection hyperplanes.
\end{abstract}

\section{INTRODUCTION}

The Hamiltonians of Gaudin's quantum integrable systems are commuting elements of the tensor power $U \mathfrak{g}^{\otimes n}$ of the universal enveloping algebra of a complex Lie algebra $\mathfrak{g}$ with a symmetric invariant tensor $t \in \operatorname{Sym}^{2}(\mathfrak{g})^{\mathfrak{g}}$. They depend on $n$ distinct complex parameters and have the form:

$$
H_{i}=H_{i}\left(z_{1}, \ldots, z_{n}\right)=\sum_{1 \leq j \leq n, j \neq i} \frac{t_{i j}}{z_{i}-z_{j}}, \quad i=1, \ldots, n .
$$

Here $t_{i j}$ is the image of the invariant tensor by the embedding $U(\mathfrak{g})^{\otimes 2} \rightarrow U(\mathfrak{g})^{\otimes n}$ placing a tensor in the $i$ th and $j$ th factor and filling with 1 . In the original context studied by Gaudin $\mathfrak{g}=s l_{2}$ and thus the $H_{i}$ act on $\left(\mathbb{C}^{2}\right)^{\otimes n}$ and can be interpreted as commuting Hamiltonians of an integrable quantum spin chain. Later the Gaudin Hamiltonians reappeared as the coefficients of the Knizhnik-Zamolodchikov flat connections $d+\kappa^{-1} \sum H_{i} d z$ on a trivial bundle on the complement of the diagonals in $\mathbb{C}^{n}$ with fiber a tensor product of $\mathfrak{g}$-modules.

The fact that the $H_{i}$ commute or, equivalently, the flatness of the KnizhnikZamolodchikov connection, follows from the Kohno-Drinfeld relations

$$
\begin{gathered}
{\left[t_{i j}, t_{k l}\right]=0, \quad \text { for distinct } i, j, k, l,} \\
{\left[t_{i j}, t_{i k}+t_{j k}\right]=0, \quad \text { for distinct } i, j, k .}
\end{gathered}
$$

The Kohno-Drinfeld Lie algebra $\mathfrak{t}_{n}$ is the quotient of the free Lie algebra in generators $t_{i j}=t_{j i}, 1 \leq i<j \leq n$ by the relations (1). It is useful to consider the Gaudin hamiltonians as commuting elements of $\mathfrak{t}_{n}$. Indeed the Kohno-Drinfeld Lie algebra maps to other relevant Lie algebras, giving rise to commuting elements there. In particular the map sending $t_{i j}$ to the permutation $(i j)$ is a homomorphism from $\mathfrak{t}_{n}$ to the group algebra of the symmetric group $S_{n}$, viewed as a Lie algebra with the commutator bracket. As the relations are homogeneous, the Kohno-Drinfeld Lie algebra comes with a grading $\oplus_{i \geq 1} \mathfrak{t}_{n}^{i}$ so that $\mathfrak{t}_{n}^{1}$ is the span of the generators. The linear span of the $H_{i}(z)$ form an $n-1$-dimensional abelian subalgebra $G_{n}(z)$

Date: 6 September 2014.

2010 Mathematics Subject Classification. Primary 81R12; Secondary 20F55, 14H70, 14N20. 
of $\mathfrak{t}_{n}$ contained in $\mathfrak{t}_{n}^{1}$, parametrized by the variety $\mathcal{M}_{0, n+1}$ of $n$-tuples of distinct complex numbers up to affine transformations. In our paper [3] we studied the closure $\mathcal{G}_{n} \subset \operatorname{Gr}\left(n-1, \mathfrak{t}_{n}^{1}\right)$ of this set of abelian subalgebras in the Grassmannian of $n$-1-planes in $\mathfrak{t}_{n}^{1}$. This closure contains in particular the span of the elements $t_{12}, t_{13}+t_{23}, \ldots, t_{1 n}+\cdots+t_{n-1, n}$, mapping to the Jucys-Murphy elements arising in the representation theory of the symmetric group.

In [3] we proved:

(i) $\mathcal{G}_{n}$ is a nonsingular projective variety isomorphic to the Deligne-Mumford moduli space $\overline{\mathcal{M}}_{0, n+1}$ of stable rational curves with $n+1$ marked points, so that $G_{n}(z)$ corresponds to $\left(\mathbb{P}^{1}, z_{1}, \ldots, z_{n}, \infty\right)$.

(ii) $\mathcal{G}_{n}$ consists of all abelian subalgebras of $\mathfrak{t}_{n}$ of maximal dimension contained in the span $\mathfrak{t}_{n}^{1}$ of generators.

The Kohno-Drinfeld Lie algebra is the special case of the $A_{n-1}$ system of a family of Lie algebras defined for general Coxeter systems (and in fact for general central arrangements of hyperplanes). The aim of this paper is to discuss the extension of our results to this more general situation. It turns out that (i) extends to general Coxeter systems but (ii) does not hold in general.

Let $\left(H_{\alpha}\right)_{\alpha \in \Delta}$ be the arrangement of complexified reflection hyperplanes $H_{\alpha} \in V$ of a finite Coxeter group, defined by a set $\Delta \in V^{*}$ of linear forms. Assume that $\Delta$ span $V^{*}$ and let $r=\operatorname{dim}(V)$. Kohno's holonomy Lie algebra $\mathfrak{t}_{\Delta}[12]$ associated with the arrangement is generated by $t_{\alpha}, \alpha \in \Delta$ with a defining relation

$$
\left[t_{\alpha}, \sum_{\beta \in W \cap \Delta} t_{\beta}\right]=0
$$

for each pair $(\alpha, W)$ consisting of a linear form $\alpha \in \Delta$ and a two-dimensional subspace $W \subset V^{*}$ containing $\alpha$. In [12] this Lie algebra appears as the Lie algebra of the unipotent completion of the fundamental group of the complement of the union of the hyperplanes of the arrangement. Its enveloping algebra is the quadratic dual of the Orlik-Solomon algebra of the arrangement in the cases where the latter is quadratic, see [20]. These relations insure the commutativity of the Gaudin Hamiltonians

$$
H(w)=\sum_{\alpha \in \Delta} \frac{\alpha(w)}{\alpha(z)} t_{\alpha}, \quad w \in V
$$

for any fixed $z$ in the complement of the arrangement. We thus obtain a family of abelian subalgebras $G_{\Delta}(z)=\operatorname{span}\{H(w), w \in V\}$ of $\mathfrak{t}_{\Delta}$ contained in the span of the generators and parametrized by the projectivization $\mathcal{M}_{\Delta}=\mathbb{P}\left(V \backslash \cup_{\alpha \in \Delta} H_{\alpha}\right)$ of the hyperplane complement.

The relations (2) and the corresponding abelian subalgebras $G_{\Delta}(z)$ arise in several different contexts:

(1) Let $\Delta$ be a set of positive roots of a simple Lie algebra $\mathfrak{g}$ with root generators $e_{\alpha}, e_{-\alpha}$ normalized so that $\left\langle e_{\alpha}, e_{-\alpha}\right\rangle=1$ for an invariant bilinear form $\langle$,$\rangle . Then t_{\alpha}=e_{\alpha} e_{-\alpha}+e_{-\alpha} e_{\alpha} \in U(\mathfrak{g})$ obey the relations (2) for any $g_{\alpha} \in U \mathfrak{h}$, the universal enveloping algebra of the Cartan subalgebra of $\mathfrak{g}$. The relations (2) (in the equivalent formulation of the commutativity of the generic subalgebras) are due to Vinberg, see [19], Theorem 2. The closure of the set of generic abelian subalgebras in the Grassmannian was described in the same paper [19] as a set in terms of certain equivalence classes of paths in the Cartan subalgebra. 
(2) The reflections $s_{\alpha}$ of a finite Coxeter group $G$ obey the relations (2). Indeed for any subspace $W \subset V^{*}$, the reflections $s_{\alpha}$ with $\alpha \in \Delta \cap W$ generate a subgroup which is also a Coxeter group and (2) is the statement that the sum of reflections of a Coxeter group is central in the group algebra, which is a consequence of the fact that the reflections form a conjugacy class. More generally we may take $t_{\alpha}=k_{\alpha} s_{\alpha}$ for a $G$-invariant function $\alpha \rightarrow k_{\alpha}$. The corresponding KZ connection $d+\sum k_{\alpha} s_{\alpha} d \log \alpha$ on the trivial bundle on the hyperplane complement with fibre $\mathbb{C} G$ was introduced by Cherednik [5].

(3) The Kohno-Drinfeld Lie algebra is the special case where $\Delta$ is the root system $A_{n-1}$. Other cases of $\mathfrak{t}_{\Delta}$ admitting a map to $U \mathfrak{g}^{\otimes n}$ for a semisimple Lie algebra $\mathfrak{g}$, include $\Delta=B_{n}$ for any $\mathfrak{g}$ and $\Delta=D_{n}$ for $\mathfrak{g}=\mathfrak{s l}_{N}$, see [13].

Define a Gaudin subalgebra of $\mathfrak{t}_{\Delta}$ to be an $r$-dimensional abelian Lie subalgebra contained in the span $\mathfrak{t}_{\Delta}^{1}$ of generators. The spans $G(z)$ of Gaudin Hamiltonians are Gaudin subalgebras and so are their limits as $z$ approaches the hyperplanes. We call these principal Gaudin subalgebras, following Vinberg. Thus the set of principal Gaudin subalgebras is the closure of the family $(G(z))_{z \in \mathcal{M}_{\Delta}}$ in the Grassmannian of $r$-planes in $\mathfrak{t}_{\Delta}^{1}$.

The main result of this paper is:

Theorem 1.1. Let $\left(H_{\alpha}\right)_{\alpha \in \Delta}$ be the arrangement of reflection hyperplanes of a Coxeter system of rank $r$. The set of principal Gaudin subalgebras is a smooth subvariety of the Grassmannian $\operatorname{Gr}\left(r, \mathfrak{t}_{\Delta}^{1}\right)$ and is isomorphic to the De ConciniProcesi compactification $\overline{\mathcal{M}}_{\Delta}$ [7] of $\mathbb{P}\left(V \backslash \cup_{\alpha \in \Delta} H_{\alpha}\right)$.

The paper is organized as follows: in Section 2 we recall the definition and description of the De Concini-Procesi compactification $\overline{\mathcal{M}}_{\Delta}$ of the complement of an arrangement of hyperplanes. In Section 3 we give an embedding of $\mathcal{M}_{\Delta}$ in the Grassmannian $\operatorname{Gr}\left(r, \mathfrak{t}_{\Delta}^{1}\right)$. These constructions apply to any central arrangement. We then specialize to the case of Coxeter arrangements and formulate Theorem 4.4 in Section 4, which is a more precise version of our result and implies Theorem 1.1.

We show that our statement does not hold for general arrangements by giving a simple counterexample, taken from $[2,1]$, where the case of general arrangement is considered. We also state a necessary and sufficient condition, due to the first author $[2,1]$, for the map $\mathcal{M}_{\Delta} \rightarrow \operatorname{Gr}\left(r, \mathfrak{t}_{\Delta}^{1}\right)$ to extend to a closed embedding of $\overline{\mathcal{M}}_{\Delta}$ in the case of a general arrangement.

A real version of the theorem also holds and leads to the moduli space tessellated by convex polyhedra known as De Concini-Procesi associahedra [7], which are generalisations of Stasheff polytopes determined by the corresponding Coxeter graphs $[4,18]$.

We conclude with the discussion of non-principal Gaudin subalgebras, using $B_{n}$ case as an example.

\section{De Concini-Procesi COMPaCtification}

Let $\left(H_{\alpha}\right)_{\alpha \in \Delta}$ be a central arrangement of hyperplanes of rank $r$, i.e., a finite family of distinct hyperplanes through the origin in an $r$-dimensional complex vector space $V$. It will be convenient to label the family by a set $\Delta \subset V^{*}$ of non-zero linear forms vanishing on the hyperplanes and work with the set of vectors $\Delta$ rather than with the hyperplanes. We assume for convenience that $\Delta$ is irreducible, in the 
sense that $V$ cannot be written as a non-trivial direct sum of two subspaces whose union contains $\Delta$. The projectivized complement $\mathcal{M}_{\Delta}=\mathbb{P}\left(V \backslash \cup_{\alpha \in \Delta} H_{\alpha}\right)$ admits a smooth compactification $\overline{\mathcal{M}}_{\Delta}$, the "wonderful model" of De Concini and Procesi.

2.1. The partially ordered set of flats. We denote by $\langle A\rangle$ the linear span of a subset $A$ of $V^{*}$ and call a non-empty subset $A \subset \Delta$ a flat if it contains all elements of $\Delta$ in its span, i.e., if $\langle A\rangle \cap \Delta=A$. The $\operatorname{rank}$ rk $A$ of a flat $A$ is the dimension of its span $\langle A\rangle$. A flat $A$ is called reducible if there are flats $A_{1}, A_{2}$ such that $A_{1} \cup A_{2}=A$ and $\langle A\rangle=\left\langle A_{1}\right\rangle \oplus\left\langle A_{2}\right\rangle$, otherwise irreducible. Irreducible flats form a partially ordered set by inclusion with maximal element $\Delta$ and minimal elements the one-point sets $\{\alpha\}, \alpha \in \Delta$.

2.2. The De Concini-Procesi compactification. If $A$ is a subset of $V^{*}$ we denote by $A^{\perp}=\cap_{\alpha \in A} H_{\alpha}$ the orthogonal complement. The natural projection $V \rightarrow V / A^{\perp}$ restricts to a map $V \backslash A^{\perp} \rightarrow V / A^{\perp} \backslash\{0\}$ and induces a projection $V \backslash A^{\perp} \rightarrow \mathbb{P}\left(V / A^{\perp}\right)$. Thus we have a map

$$
j_{\Delta}: \mathcal{M}_{\Delta} \hookrightarrow \prod_{A} \mathbb{P}\left(V / A^{\perp}\right)
$$

with product taken over all irreducible flats. The map is injective since $A=\Delta$ is irreducible and thus $\mathbb{P}\left(V / \Delta^{\perp}\right)=\mathbb{P}(V)$ belongs to the product.

The De Concini-Procesi compactification $\overline{\mathcal{M}}_{\Delta}$ is by definition the closure of the image of $j_{\Delta}$. It is a smooth projective variety containing $j_{\Delta}\left(\mathcal{M}_{\Delta}\right) \cong \mathcal{M}_{\Delta}$ as a Zariski open subset whose complement is the union of smooth divisors with normal crossings [7].

2.3. Nested sets. A nested set for $\Delta$ is a set $S$ of irreducible flats of $\Delta$ so that for any subset $\left\{A_{1}, \ldots, A_{k}\right\}$ of $S$ consisting of pairwise non-comparable flats, we have $\left\langle\cup_{i=1}^{k} A_{i}\right\rangle=\left\langle A_{1}\right\rangle \oplus \cdots \oplus\left\langle A_{k}\right\rangle$. Nested sets are partially ordered by inclusion.

Lemma 2.1. ([7], p. 500) Let $S$ be a nested set containing $\Delta$ and $\alpha \in \Delta$. Then the subsets of $A$ containing $\alpha$ are linearly ordered. Thus there is a unique minimal flat $A_{S}(\alpha) \in S$ such that $\alpha \in A$.

We are particularly interested in maximal nested sets. They have the property ([7], Proposition 1.1) that for every $A \in S$ the sets $A_{1}, \ldots, A_{k} \in S$ that are maximal proper subsets of $A$ obey $\sum \operatorname{dim}\left(A_{i}\right)=\operatorname{dim}(A)-1$. Thus one can pick a vector $\alpha_{A} \in A \backslash \cup_{i} A_{i}$ and obtain a basis of $V$ labeled by $S$. Such bases are called adapted bases for the maximal nested set $S$. They have the property that their intersection with any $A \in S$ is a basis of $\langle A\rangle$.

2.4. An open cover of $\overline{\mathcal{M}}_{\Delta}$. The De Concini-Procesi variety $\overline{\mathcal{M}}_{\Delta}$ admits a cover by open affine subsets $U_{S}$ labeled by maximal nested sets $S$. For every maximal nested set $S$ the open subset $U_{S}$ is defined as the set of $z \in \overline{\mathcal{M}}_{\Delta}$ such that for every $A \in S$ the projection $z_{A} \in \mathbb{P}\left(V / A^{\perp}\right)$ obeys

$$
\forall \alpha \in A \backslash \cup_{i=1}^{k} A_{i}: \alpha\left(z_{A}\right) \neq 0,
$$

where $A_{1}, \ldots, A_{k} \in S$ are the maximal elements properly contained in $A$.

Theorem 2.2. (De Concini-Procesi, [7] Sec. 1.1)

(i) The sets $U_{S}$, where $S$ runs over the maximal nested sets for $\Delta$, form an open covering of $\overline{\mathcal{M}}_{\Delta}$.

(ii) The natural projection $U_{S} \rightarrow \prod_{A \in S} \mathbb{P}\left(V / A^{\perp}\right)$ is a closed embedding. 


\section{A MAP TO THE GRASSMANNIAN}

Let $\Delta$ be a set of non-zero, pairwise non-collinear vectors in an $r$-dimensional complex vector space $V$. Kohno's holonomy Lie algebra $\mathfrak{t}_{\Delta}$ is the graded Lie algebra with generators $t_{\alpha}$ of degree 1 labeled by $\alpha \in \Delta$ and relations (2). Let $\mathfrak{t}_{\Delta}^{1}$ be span of generators. For our present purpose it is just a vector space with basis $\left(t_{\alpha}\right)_{\alpha \in \Delta}$. We construct a map from $\overline{\mathcal{M}}_{\Delta}$ to the Grassmannian $\operatorname{Gr}\left(r, \mathfrak{t}_{\Delta}^{1}\right)$ of $r$-dimensional subspaces of $\mathfrak{t}_{\Delta}^{1}$ restricting to a locally closed embedding of $\mathcal{M}_{\Delta}$. The uncompactified $\mathcal{M}_{\Delta}$ is embedded by sending a point in the hyperplane complement to the span of the corresponding Gaudin Hamiltonians:

$$
\begin{aligned}
i: \mathcal{M}_{\Delta} & =\mathbb{P}\left(V \backslash \cup_{\alpha \in \Delta} H_{\alpha}\right) \rightarrow \operatorname{Gr}\left(r, \mathfrak{t}_{\Delta}^{1}\right) \\
z & \mapsto\left\{\sum_{\alpha \in \Delta} \frac{\alpha(w)}{\alpha(z)} t_{\alpha}, w \in V\right\} .
\end{aligned}
$$

Proposition 3.1. The embedding $i: \mathcal{M}_{\Delta} \rightarrow \operatorname{Gr}\left(r, \mathfrak{t}_{\Delta}^{1}\right)$ extends uniquely to a map $\bar{i}: \overline{\mathcal{M}}_{\Delta} \rightarrow \operatorname{Gr}\left(r, \mathfrak{t}_{\Delta}^{1}\right)$.

The uniqueness is obvious since $\mathcal{M}_{\Delta}$ is a Zariski open subset. We prove the existence by analyzing the map $i$ on each of the open sets $U_{S}$ labeled by the maximal nested sets $S$. We also prove some further properties of $\left.i\right|_{U_{S}}$ that will be useful to prove that $\bar{i}$ is an embedding in the case of Coxeter systems.

Let $S$ be a nested set for $\Delta$ and let $B=\left(\alpha_{A}\right)_{A \in S}$ be an adapted basis for $S$. Let $V_{B}$ be the open subset of $\operatorname{Gr}\left(r, \mathfrak{t}_{\Delta}^{1}\right)$ consisting of $r$-planes whose projection onto the span of $t_{\beta}$ with $\beta \in B$ is surjective. Such $r$-planes are given by systems of equations of the form

$$
t_{\alpha}^{*}-\sum_{\beta \in B} c_{\alpha, \beta} t_{\beta}^{*}=0, \quad \alpha \in \Delta \backslash B .
$$

where $t_{\alpha}^{*}$ is the basis of $\left(\mathfrak{t}_{\Delta}^{1}\right)^{*}$ dual to $t_{\alpha}$ and $c_{\alpha, \beta}$ are arbitrary scalar coefficients. Let $\operatorname{supp}_{B}(\alpha) \subset B$ be the set of basis elements occurring with nonzero coefficient in the expression of $\alpha \in \Delta$ as linear combination of $B$ and let $V_{B}^{0}$ be the subspace of the affine space $V_{B}$ given by the equations

$$
c_{\alpha, \beta}=0, \quad \text { if } \beta \notin \operatorname{supp}_{B}(\alpha) .
$$

Let $\alpha=\sum_{\beta \in B} n_{\alpha, \beta} \beta$ be the expression of $\alpha$ as linear combination of the basis. Then for $z=\left(z_{A}\right)_{A \in \Delta}$ we set

$$
i_{S}(z)=\cap_{\alpha \in \Delta \backslash B} \operatorname{Ker}\left(\alpha\left(z_{A_{S}(\alpha)}\right) t_{\alpha}^{*}-\sum_{\beta \in B} n_{\alpha, \beta} \beta\left(z_{A_{S}(\alpha)}\right) t_{\beta}^{*}\right) \subset V .
$$

Here $A_{S}(\alpha)$ denotes the smallest $A \in S$ containing $\alpha$ (cf. Lemma 2.1).

Lemma 3.2. Let $S$ be a maximal nested set for $\Delta$.

(i) For all $z \in U_{S}, i_{S}(z) \in V_{B}^{0}$.

(ii) The map $i_{S}$ coincides with $i$ on $U_{S} \cap \mathcal{M}_{\Delta}$.

Proof. (i) To show that $i_{S}\left(U_{S}\right) \subset V_{B}$ we need to show that $\alpha\left(z_{A_{S}(\alpha)}\right) \neq 0$ for $z \in U_{S}$. By construction, $\alpha \in A_{S}(\alpha)$ and $\alpha$ is not contained in any other $A \in S$ contained in $A_{S}(\alpha)$. Thus, by definition of $U_{S}, \alpha\left(z_{A_{S}(\alpha)}\right) \neq 0$. It is clear that the coefficients $c_{\alpha, \beta}$ in the image of $i_{S}$ obey (4). 
(ii) If $\left(z_{A}\right)_{A \in \Delta} \in \mathcal{M}_{\Delta}$ then $z_{A}$ is the image of $z \in V \backslash \cup_{\alpha} H_{\alpha}$ and $i_{S}(z)$ is an $r$ dimensional subspace containing the Gaudin Hamiltonians $H(w)=\sum_{\gamma \in \Delta} \frac{\gamma(w)}{\gamma(z)} t_{\gamma}$, $w \in V$. Indeed

$$
\left(\alpha(z) t_{\alpha}^{*}-\sum_{\beta \in B} n_{\alpha, \beta} \beta(z) t_{\beta}^{*}\right)(H(w))=\alpha(w)-\sum_{\beta} n_{\alpha, \beta} \beta(w)=0 .
$$

Since the Gaudin Hamiltonians form an $r$-dimensional vector space, their span coincides with the image of $i_{S}$.

The maps $i_{S}$ are thus the restrictions of a map $\bar{i}: \overline{\mathcal{M}}_{\Delta} \rightarrow \operatorname{Gr}\left(r, \mathfrak{t}_{\Delta}^{1}\right)$ coinciding with $i$ on $\mathcal{M}_{\Delta}$, proving Proposition 3.1.

Lemma 3.3. The r-planes in the image of $\bar{i}: \overline{\mathcal{M}}_{\Delta} \rightarrow \operatorname{Gr}\left(r, \mathfrak{t}_{\Delta}^{1}\right)$ all contain the vector $C_{\Delta}=\sum_{\alpha \in \Delta} t_{\alpha}$.

Proof.

$$
\left(\alpha(z) t_{\alpha}^{*}-\sum_{\beta \in B} n_{\alpha, \beta} \beta(z) t_{\beta}^{*}\right)\left(C_{\Delta}\right)=\alpha(z)-\sum_{\beta} n_{\alpha, \beta} \beta(z)=0
$$

Remark 3.4. The vector $C_{\Delta}$ spans the center of the holonomy algebra $\mathfrak{t}_{\Delta}$.

\section{The CASE of CoXeter Systems}

Let us consider the special case of the arrangement of reflection hyperplanes of an irreducible Coxeter group $G$. In this case we can describe maximally nested sets combinatorially in terms of subsets of the set of nodes of the Coxeter diagrams. We start by recalling some facts about Coxeter systems and explain the properties we will need.

4.1. Coxeter root systems. We follow [10]. Let $E$ be an $r$-dimensional Euclidean vector space and for $\alpha \in E \backslash\{0\}$ denote by $s_{\alpha}$ the orthogonal reflection with respect to the hyperplane with normal vector $\alpha$. A (Coxeter) root system is a finite set $\Phi$ of nonzero vectors in $E$, called roots, such that

(1) For all $\alpha \in \Phi, \mathbb{R} \alpha \cap \Phi=\{\alpha,-\alpha\}$

(2) For all $\alpha \in \Phi, s_{\alpha} \Phi=\Phi$.

A root system is called reducible if $E$ is the orthogonal direct sum of two subspaces of positive dimension whose union contains $\Phi$, otherwise irreducible. The group $G$ generated by the reflections $s_{\alpha}, \alpha \in \Phi$ is then a finite Coxeter group and any finite Coxeter group is of this form. Any linear form that does not vanish on roots decomposes $\Phi$ into positive and negative roots, according to the sign it takes on roots. There is then a unique basis of $E$ consisting of roots with the property that all positive roots are linear combinations of basis vectors with nonnegative coefficients. Such a basis is called basis of simple roots and the corresponding reflections simple reflections. The Coxeter group acts simply transitively on bases of simple roots. Angles between simple roots are of the form $\pi(1-1 / m)$ with $m \in \mathbb{Z}_{\geq 2}$. These data are encoded in the Coxeter graph, a graph with labeled edges with simple roots as vertices and an edge with label $m$ connecting non-orthogonal simple roots making an angle $\pi(1-1 / m),(m \geq 3)$. Irreducible root systems have connected Coxeter graphs. 
Lemma 4.1. Let $\Phi \subset E$ be an irreducible Coxeter systems and $B$ a basis of simple roots. Then there is root $\theta \in \Phi$ which is a linear combination of simple roots with positive coefficients.

Proof. Let us order the simple roots $B=\left\{\alpha_{1}, \ldots, \alpha_{r}\right\}$ so that the subgraphs with vertices $\alpha_{1}, \ldots, \alpha_{i}$ are connected for all $i=1, \ldots, r$. This is clearly possible since the Coxeter graph is connected. This condition means that for each $i>1$ there is a $j<i$ such that the inner product $\left(\alpha_{i}, \alpha_{j}\right)$ is non-zero and in fact (since all angles are obtuse) negative. Let $s_{i}$ denote the corresponding simple reflections. We claim that, for all $i, v_{i}:=s_{i} \cdots s_{3} s_{2} \alpha_{1}$ is a linear combination of $\alpha_{1}, \ldots, \alpha_{i}$ with positive coefficients. This is obvious for $i=1$. Assuming by induction that $v_{i}$ has the required property, we see that in

$$
v_{i+1}=s_{i+1} v_{i}=v_{i}-2 \frac{\left(\alpha_{i+1}, v_{i}\right)}{\left(\alpha_{i+1}, \alpha_{i+1}\right)} \alpha_{i+1}
$$

the inner product $\left(\alpha_{i+1}, v_{i}\right)$ is negative, proving the induction step. Thus we may take $\theta=s_{r} \cdots s_{2} \alpha_{1}$.

4.2. De Concini-Procesi compactification for Coxeter arrangements. Let $\Phi \subset E$ be a root system and $G$ the corresponding group generated by reflections. Fix a basis $B$ of simple roots and let $\Delta=\Phi_{+}$be the corresponding set of positive roots. We view $\Delta$ as a subset of the dual of the complexification of $E \simeq E^{*}$, so that the complexified reflection hyperplanes $H_{\alpha} \subset V$ are the kernels of $\alpha \in \Delta$. Then $G$ acts on the set of hyperplanes and thus on the set of nested sets. We say that two nested sets are $G$-equivalent if they are related by an element of $G$.

Proposition 4.2. Let $\Phi \subset V^{*}$ be an irreducible root system with Coxeter group $G$. Fix a basis $B$ of simple roots. Then every nested set $S$ is $G$-equivalent to one where each subset in $S$ is spanned by simple roots. Thus, up to $G$-equivalence, every maximal nested set has an adapted basis consisting of simple roots.

This proposition is proved in [7], Sec. 3.1, in the case of Weyl groups. The same proof applies to the Coxeter case.

Remark 4.3. A consequence of Proposition 4.2 is that, up to $G$-equivalence, maximal nested sets for Coxeter arrangement are obtained from maximal nested sets on Coxeter graphs, whose vertices are naturally labeled by simple roots. By definition, a nested set on a graph $\Gamma$ is a set of nonempty connected subgraphs of $\Gamma$ which are pairwise either disjoint or contained one into the other. Here two subgraphs are called disjoint if there are no edges joining vertices from these two subgraphs. To a connected subgraph $I$ of $\Gamma$ one associates the flat generated by simple roots labelling the vertices of $I$.

Theorem 4.4. Let $\left(H_{\alpha}\right)_{\alpha \in \Delta}$ be the set of reflection hyperplanes of a Coxeter system of rank $r$. Then the map $\bar{i}: \overline{\mathcal{M}}_{\Delta} \rightarrow \operatorname{Gr}\left(r, \mathfrak{t}_{\Delta}^{1}\right)$ is a closed embedding.

Proof. It is sufficient to consider the case of irreducible Coxeter root systems. By Lemma 3.3 the image of $\bar{i}$ is contained in the subvariety $G^{\prime}$ of $r$-planes containing $C_{\Delta}$. It is sufficient to show that for each nested set $S$ we can choose an adapted basis $B$ so that $\bar{i}: U_{S} \rightarrow V_{B}^{0} \cap G^{\prime}$ has a left inverse $\varphi_{S}: V_{B}^{0} \cap G^{\prime} \rightarrow U_{S}$, i.e., a regular map such that $\varphi_{S} \circ i=\mathrm{id}_{U_{S}}$. The subset $V_{B}^{0}$ is the subspace of the affine space $V_{B}$ 
consisting of $r$-dimensional subspaces determined by equations of the form

$$
t_{\alpha}^{*}-\sum_{\beta \in \operatorname{supp}_{B}(\alpha)} c_{\alpha, \beta} t_{\beta}^{*}=0, \quad \alpha \in \Delta \backslash B .
$$

It has coordinates $c_{\alpha, \beta}, \beta \in \operatorname{supp}_{B}(\alpha)$ and $V_{B}^{0} \cap G^{\prime}$ is the affine subspace of $V_{B}^{0}$ given by the equations

$$
\sum_{\beta \in \operatorname{supp}_{A_{S}}(\alpha)} c_{\alpha, \beta}=1, \quad \alpha \in \Delta \backslash B
$$

Let us view $U_{S}$ as a subset of $\prod_{A \in S} \mathbb{P}\left(V / A^{\perp}\right)$ via Theorem 2.2 (ii). The coordinates of $i(z)$ are given in terms of the projections $z_{A} \in \mathbb{P}\left(V / A^{\perp}\right)$ of $z \in U_{S}$ by

$$
c_{\alpha, \beta}=n_{\alpha, \beta} \frac{\beta\left(z_{A}\right)}{\alpha\left(z_{A}\right)}, \quad \alpha \in \Delta \backslash B, \quad \beta \in B .
$$

Here $A=A_{S}(\alpha)$ is the smallest $A \in S$ containing $\alpha$, so that, by definition of $U_{S}$, the denominator does not vanish. Pick for every $A \in S$ a root $\theta=\theta_{A} \in A$ with $n_{\theta, \beta} \neq 0$ for all $\beta \in B \cap A$. Such a root exists by Lemma 4.1. In particular $A$ is the smallest set in $S$ containing $\theta_{A}$. For any $W \in V_{B} \cap G^{\prime}$ with coordinates $\left(c_{\alpha, \beta}\right)$ define the component $z_{A}$ of $z=\varphi(W)$ as the line through $\tilde{z}_{A} \in V / A^{\perp}$ such that

$$
\beta\left(\tilde{z}_{A}\right)=\frac{c_{\theta, \beta}}{n_{\theta, \beta}}, \quad \theta=\theta_{A}, \quad \beta \in B \cap A
$$

As $B \cap A$ is a basis of $\left(V / A^{\perp}\right)^{*}$ this uniquely defines a vector $\tilde{z}_{A} \in V / A^{\perp}$. By (5) it is normalized by $\theta\left(\tilde{z}_{A}\right)=1$. In particular $\tilde{z}_{A}$ is nonzero and defines a line $z_{A} \in \mathbb{P}\left(V / A^{\perp}\right)$ obeying (6) for $\alpha=\theta$. Repeating this for all $A \in S$ we obtain a map

$$
\varphi_{S}: V_{B} \rightarrow \prod_{A \in S} \mathbb{P}\left(V / A^{\perp}\right)
$$

By construction $\varphi_{S} \circ i=\operatorname{id}_{U_{S}}$.

One may ask whether the map $\bar{i}$ is a closed embedding for general arrangement $\Delta$. This is not the case. The following counterexample is taken from $[2,1]$. Let $V^{*}$ have a basis $\left\{\alpha_{1}, \alpha_{2}, \alpha_{3}\right\}$ and let $\Delta=\left\{\alpha_{1}, \alpha_{2}, \alpha_{3}, \alpha_{1}+\alpha_{2}, \alpha_{1}+\alpha_{3}\right\}$. The projectivized arrangement of hyperplanes is then given by five lines in the projective plane forming the sides and a diagonal of a quadrilateral. There are exactly two points $P, Q$ at which three lines meet. The De Concini-Procesi compactification $\overline{\mathcal{M}}_{\Delta}$ is the blowup $\widehat{\mathbb{P}^{2}}$ of $\mathbb{P}^{2}$ at these two points. It surjects under $\bar{i}$ to the closure $\overline{i\left(\mathcal{M}_{\Delta}\right)}$ in the Grassmannian of the span of Gaudin Hamiltonians, which is isomorphic to $\mathbb{P}^{1} \times \mathbb{P}^{1}:\left(\lambda_{1}: \lambda_{2}, \mu_{1}: \mu_{2}\right) \in \mathbb{P}^{1} \times \mathbb{P}^{1}$ corresponds to the Gaudin subalgebra $\operatorname{span}\left(C_{\Delta}, \lambda_{1} t_{\alpha_{2}}+\lambda_{2} t_{\alpha_{1}+\alpha_{2}}, \mu_{1} t_{\alpha_{3}}+\mu_{2} t_{\alpha_{1}+\alpha_{3}}\right)$. We have the diagram of birational morphisms

$$
\mathbb{P}^{2} \leftarrow \overline{\mathcal{M}}_{\Delta} \cong \widehat{\mathbb{P}^{2}} \stackrel{\bar{i}}{\rightarrow} \overline{i\left(\mathcal{M}_{\Delta}\right)} \cong \mathbb{P}^{1} \times \mathbb{P}^{1}
$$

The map $\bar{i}$ is the blowdown of the proper transform of the line through $P$ and $Q$. It maps a curve to a point and is therefore not injective.

In the first author's Ph.D. thesis, the following necessary and sufficient condition for the map $\bar{i}$ to be a closed embedding is proved (see 2.1 for the terminology). 
Theorem 4.5. (L. Aguirre $[2,1])$ Let $\left(H_{\alpha}\right)_{\alpha \in \Delta}$ be a general central arrangement of hyperplanes as in Section 2. The map $\bar{i}: \overline{\mathcal{M}}_{\Delta} \rightarrow \operatorname{Gr}\left(r, \mathfrak{t}_{\Delta}^{1}\right)$ is a closed embedding if and only if $\Delta$ has the following property. For any irreducible flat $A \in \Delta$ and any flat $F<A$ of rank $\mathrm{rk} A-1$, there is no proper subflat $K<F$ such that

$$
\langle A \backslash F\rangle \cap\langle F\rangle \subset\langle K\rangle .
$$

\section{REAL VERSION AND GRAPH-ASSOCIAHEDRA}

All the considerations above work over reals as well. The corresponding real wonderful models $\bar{M}_{\Delta}(\mathbb{R})$ were studied in the $A_{n-1}$ case by Kapranov [11] and in the general Coxeter case by De Concini and Procesi [7] (see also Gaiffi [9]).

They can be described as an iterated real blow-up of the projective space and can be glued from $|G| / 2$ copies of the following generalisations of Stasheff polytope, or associahedron. The corresponding convex polytopes are known as graphassociahedra [4], or De Concini-Procesi associahedra [18]. They have the following explicit description $[8,18]$ inspired by $[14,17]$.

Let $\Gamma$ be a connected graph with set of vertices $B$ and consider the set $S_{\Gamma}$ of all connected subgraphs $I \subset \Gamma$ excluding $\Gamma$. The corresponding graph-associahedra can be defined as the following convex polytope in $\mathbb{R}^{|B|}$ with coordinates labeled by the vertices of $\Gamma$

$$
P_{\Gamma}=\left\{x \in \mathbb{R}^{|B|}: \sum_{\alpha \in B} x_{\alpha}=3^{|B|}, \sum_{\alpha \in I} x_{\alpha} \geq 3^{|I|}, I \in S_{\Gamma}\right\} .
$$

It is known $[4,18]$ that $P_{\Gamma}$ is a simple, convex polytope, whose face poset is isomorphic to the poset of the nested sets on $\Gamma$ (see the definition in remark 4.3). In particular, the vertices of $\Gamma$ corresponds to the maximal nested sets. One can also describe it as the iterated truncation of a simplex [4].

In the case when $\Gamma$ is a path with $r$ vertices this gives a particular realisation of the Stasheff polytope $K_{r+1}$, which was initially introduced only combinatorially $[16]$.
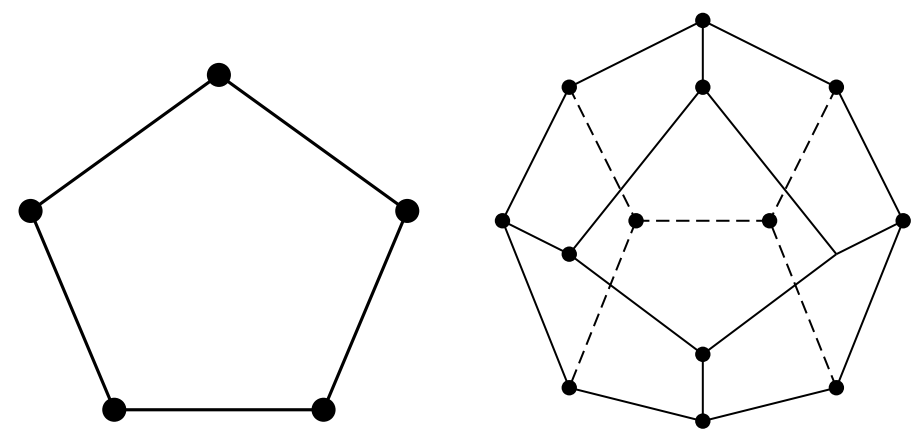

Figure 1. Stasheff polytopes $K_{4}$ and $K_{5}$.

When $\Gamma=\Gamma_{G}$ is a Coxeter graph we have the De Concini-Procesi associahedron $P_{G}$.

Note that the labelling of the angles on the Coxeter graph does not affect the definition of the polytope, so the polytopes $P_{G}$ for the arrangements of types $A_{n}$ and $B_{n}=C_{n}$ are the same Stasheff polytopes $K_{n+1}$. 
The space $\bar{M}_{\Delta}(\mathbb{R})$ is tessellated by $|G| / 2$ copies of $P_{G}$, which is isomorphic to the closure of the real points corresponding to one Coxeter chamber [7]. The details of the gluing can be found in [4]. In particular, in the $A_{3}$ and $B_{3}$ cases we have two different non-oriented surfaces glued from 12 and 24 pentagons, which topologically are the connected sums of 5 and 8 copies of the real projective plane, respectively.

As it follows from our results modulo this gluing one can use $P_{G}$ as a geometric chart of all principal Gaudin subalgebras in the real case (see the discussion of the $A_{n}$ case in [15]).

\section{NON-PRINCIPAL GAUdin SUBALGEBRAS}

It is natural to ask if there are Gaudin subalgebras outside the principal family. We have shown in [3] that in the $A_{n}$ case the answer is negative. However, already in the $B_{n}$ case not all Gaudin subalgebras are principal.

Let

$$
r_{i}, t_{i j}=t_{j i}, s_{i j}=s_{j i}, 1 \leq i \leq j \leq n
$$

be the generators of the $B_{n}$ holonomy Lie algebra, corresponding to the hyperplanes $z_{i}=0, z_{i}-z_{j}=0, z_{i}+z_{j}=0$, respectively. The defining relations have the form for pairwise distinct $i, j, k, l$

$$
\begin{aligned}
& {\left[r_{i}, r_{j}+t_{i j}+s_{i j}\right]=0,} \\
& {\left[t_{i j}, r_{i}+r_{j}+s_{i j}\right]=0,} \\
& {\left[s_{i j}, r_{i}+r_{j}+t_{i j}\right]=0}
\end{aligned}
$$

for $B_{2}$ subsystems,

$$
\begin{aligned}
{\left[t_{i j}, t_{i k}+t_{j k}\right] } & =0 \\
{\left[t_{i j}, s_{i k}+s_{j k}\right] } & =0 \\
{\left[s_{i j}, t_{i k}+s_{j k}\right] } & =0
\end{aligned}
$$

for $A_{2}$ subsystems, and

$$
\begin{gathered}
{\left[r_{i}, t_{j k}\right]=\left[r_{i}, s_{j k}\right]=0} \\
{\left[t_{i j}, t_{k l}\right]=\left[t_{i j}, s_{k l}\right]=\left[s_{i j}, s_{k l}\right]=0}
\end{gathered}
$$

for $A_{1} \times A_{1}$ subsystems.

The principal family of Gaudin subalgebras is the closure of the family

$$
G\left(z_{1}, \ldots, z_{n}\right)=\left\{\sum_{i=1}^{n} \frac{a_{i}}{z_{i}} r_{i}+\sum_{i<j}^{n} \frac{a_{i}-a_{j}}{z_{i}-z_{j}} t_{i j}+\sum_{i<j}^{n} \frac{a_{i}+a_{j}}{z_{i}+z_{j}} s_{i j}, a \in \mathbb{C}^{n}\right\},
$$

but it is easy to see that there is another family of Gaudin subalgebras

$$
G^{A}\left(z_{1}, \ldots, z_{n}\right)=\left\{\sum_{i=1}^{n} \frac{a_{i}}{z_{i}} r_{i}+\sum_{i<j}^{n} \frac{a_{i}-a_{j}}{z_{i}-z_{j}}\left(t_{i j}+s_{i j}\right), a \in \mathbb{C}^{n}\right\} .
$$

The closure of the second family is isomorphic to the $A_{n}$-type wonderful model, which is the Deligne-Mumford-Knudsen moduli space $\bar{M}_{0, n+2}$.

So the variety of $B_{n}$-Gaudin subalgebras contains at least two $(n-1)$-dimensional subvarieties. For $n \geq 3$ they are irreducible components but they are not the only ones, as explained in [1]. We close by examining the cases $n=2$ and $n=3$. Details can be found in [2].

The $B_{2}$-Case: The variety of Gaudin subalgebras is a non-singular irreducible subvariety of $\operatorname{Gr}\left(2, \mathfrak{t}_{\Delta}^{1}\right)$ isomorphic to $\mathbb{P}^{2}$. Indeed, in that case any 2-dimensional 
subspace of $\mathfrak{t}^{1}$ containing the central element $c=r_{1}+r_{2}+t_{12}+s_{12}$ is a Gaudin subalgebra. The closures of the families (7) and (8) are $\mathbb{P}^{1}$-subvarieties of it. One can check that the first one is a conic, the second one is a line, intersecting in two points, which are $\operatorname{span}\left(c, r_{1}\right)$ and $\operatorname{span}\left(c, r_{2}\right)$.

The $B_{3}$-Case: The variety of Gaudin subalgebras is comprised of eight nonsingular irreducible components of different dimensions. Among them are the twodimensional closures of the families (7) and (8), which we denote by $B_{\{123\}}$ and $A_{\{123\}}$ respectively. The first one is the special case of weak del Pezzo surface of degree 2 isomorphic to the double cover of $\mathbb{P}^{2}$ branched over configuration of 4 lines in generic position, ${ }^{1}$ while the second one is the degree 5 del Pezzo surface, which is $\mathbb{P}^{2}$ blown up at 4 points in generic position.

Three additional two-dimensional irreducible components correspond to the $B_{2}$ subsystems and each is isomorphic to $\mathbb{P}^{2}$. We call them $B_{\{12\}}, B_{\{13\}}$ and $B_{\{23\}}$. The Gaudin subalgebra corresponding to a point $\left(x_{i}: x_{j}: x_{i j}\right) \in \mathbb{P}^{2} \cong B_{\{i j\}}$, for $1 \leq i<j \leq 3$, is of the form

$$
\operatorname{span}\left(c_{\Delta}, c_{i j}, x_{i} r_{i}+x_{j} r_{j}+x_{i j} t_{i j}\right)
$$

where $c_{\Delta}$ is the central element

$$
c_{\Delta}=\sum_{i=1}^{3} r_{i}+\sum_{i<j}^{3}\left(t_{i j}+s_{i j}\right)
$$

and

$$
c_{i j}=r_{i}+r_{j}+t_{i j}+s_{i j} .
$$

Finally there are three $\mathbb{P}^{1}$-components which we call $A_{\{12\}}, A_{\{13\}}$ and $A_{\{23\}}$. The Gaudin subalgebra corresponding to a point $\left(y_{i}: y_{j}\right) \in \mathbb{P}^{1} \cong A_{\{i j\}}$ is of the form

$$
\operatorname{span}\left(c_{\Delta}, r_{1}+r_{2}+r_{3}+t_{i j}+s_{i j}, y_{i} t_{i j}+y_{j} s_{i j}\right) .
$$

The irreducible components of the Gaudin variety intersect as follows: $B_{\{123\}}$ and $A_{\{123\}}$ each intersect $B_{\{i j\}}$ in a respective $\mathbb{P}^{1}$-curve. Those two curves intersect at two points

$$
\operatorname{span}\left(c_{\Delta}, c_{i j}, r_{i}\right), \operatorname{span}\left(c_{\Delta}, c_{i j}, r_{j}\right)
$$

giving altogether six points, which are the only points of intersection of the components $B_{\{123\}}$ and $A_{\{123\}}$. Each of the curves $A_{\{i j\}}$ does not meet the component $B_{\{123\}}$ and intersects $A_{\{123\}}$ at a single point

$$
\operatorname{span}\left(c_{\Delta}, r_{1}+r_{2}+r_{3}, t_{i j}+s_{i j}\right) .
$$

Notice that for the $B_{3}$-case, the variety of Gaudin subalgebras contains as components the Gaudin varieties of types $A_{l}$ and $B_{l}$ for any $l<3$. This result can be extended to the $B_{r}$-case where, apart from an irreducible $B_{r}$ - and $A_{r}$-component, all Gaudin varieties of types $A_{l}$ and $B_{l}, l<r$ are contained (in multiple copies). The intersection structure of these subvarieties is quite intricate, but its combinatorics could in principle be deduced from the intersection lattice in a recursive manner. It is not completely clear whether one is able to capture all Gaudin subalgebras in this recursive way for $r>3$.

It is however remarkable that for $B_{n}$ there are no abelian subalgebras contained in $\mathfrak{t}_{\Delta}^{1}$ which have dimensions larger than $n$, i.e., Gaudin subalgebras are maximal exactly as in the $A_{n}$-case. This fact is owed to the arrangement being fibre-type. It

\footnotetext{
${ }^{1}$ We are grateful to Artie Prendergast-Smith, who explained this to us.
} 
is known that among the finite Coxeter arrangements only the $A_{n}, B_{n}$ and dihedral cases are of fibre-type and indeed in every other case there can be found abelian subalgebras of higher dimension than the rank of the arrangement (see [2]).

The analysis of the remaining Coxeter arrangements [2] shows that the only case when all Gaudin subalgebras are principal is $A_{n}$, which thus turns out to be very exceptional.

\section{ACKNowledgements.}

We are grateful to I. Cherednik, M. Kapranov, T. Kohno and A. PrendergastSmith for helpful and stimulating discussions.

The work of APV was partly supported by the EPSRC (grant EP/J00488X/1). The work of GF was partly supported by the Swiss National Science Foundation (National Centre of Competence in Research "The Mathematics of Physics - SwissMAP").

\section{REFERENCES}

[1] L. Aguirre, The variety of principal Gaudin subalgebras for an arbitrary hyperplane arrangement, in preparation.

[2] L. Aguirre, On the notion of Gaudin subalgebras for general hyperplane arrangements. PhD Thesis 21880, ETH Zurich (2014). http://dx.doi.org/10.3929/ethz-a-010294025

[3] L. Aguirre, G. Felder and A.P. Veselov, Gaudin subalgebras and stable rational curves. Compos. Math. 147 (2011), no. 5, 1463-1478.

[4] M. Carr and S. L. Devadoss, Coxeter complexes and graph-associahedra. Topology and its Applications, 153, Issue 12 (2006), 2155-2168.

[5] I. Cherednik, Monodromy representations for generalized Knizhnik-Zamolodchikov equations and Hecke algebras. Publ. Res. Inst. Math. Sci. 27 (1991), no. 5, 711-726.

[6] C. De Concini and C. Procesi, Wonderful models of subspace arrangements, Selecta Math. (N.S.) 1 (1995), 459-494.

[7] C. De Concini and C. Procesi, Hyperplane Arrangements and Holonomy Equations. Selecta Mathematica, New Series Vol. 1, No. 3 (1995); 495-535.

[8] S. L. Devadoss, A realization of graph associahedra. Discrete Mathematics, 309, Issue 1, 2009, 271-276.

[9] G. Gaiffi, Real structures of models of arrangements. Int. Math. Res. Not. 2004 (64) (2004), $3439-3467$.

[10] J. E. Humphreys, Reflection groups and Coxeter groups. Cambridge University Press, 1990.

[11] M.M. Kapranov The permutoassociahedron, Mac Lane's coherence theorem and asymptotic zones for the KZ equation. J. Pure Appl. Algebra 85 (1993), no. 2, 119-142.

[12] T. Kohno, Holonomy Lie algebras, logarithmic connections and the lower central series of fundamental groups. Singularities (Iowa City, IA, 1986), 171-182, Contemp. Math. 90, Amer. Math. Soc., Providence, RI, 1989.

[13] A. Leibman, Some monodromy representations of generalized braid groups. Comm. Math. Phys. 164 (1994), no. 2, 293-304.

[14] M. Markl, Simplex, associahedron, and cyclohedron, Higher homotopy structures in topology and mathematical physics (Poughkeepsie, NY, 1996), 235-265, Contemp. Math. 227, AMS, 1999.

[15] K. Schöbel, A.P. Veselov, Separation coordinates, moduli spaces and Stasheff polytopes. Comm. Math. Phys. 337 (2015), 1255-1274.

[16] J. Stasheff, Homotopy associativity of $H$-spaces. I, II. Trans. Amer. Math. Soc. 108 (1963), $275-312$.

[17] J. D. Stasheff, From operads to physically inspired theories, Operads: Proceedings of Renaissance Conferences (Hartford, CT/Luminy, 1995), 53-81, Contemp. Math. 202, AMS, 1997.

[18] V. Toledano-Laredo, Quasi-Coxeter algebras, Dynkin diagram cohomology and quantum Weyl groups. International Mathematics Research Papers 2008, article ID rpn009, 167 pages. 
[19] E. B. Vinberg, Some commutative subalgebras of a universal enveloping algebra. Izv. Akad. Nauk SSSR Ser. Mat. 54 (1990), no. 1, 3-25, 221; translation in Math. USSR-Izv. 36 (1991), no. $1,1-22$

[20] S. A. Yuzvinskii, Orlik-Solomon algebras in algebra and topology Uspekhi Mat. Nauk 56 (2001), no. 2(338), 87-166; translation in Russian Math. Surveys 56 (2001), no. 2, 293-364

Department of mathematics, ETH Zurich, 8092 Zurich, Switzerland

Department of Mathematics, ETH Zurich, 8092 Zurich, Switzerland

Department of Mathematical Sciences, Loughborough University, Loughborough LE11 3TU, UK and Moscow State University, Moscow 119991, Russia 